ELECTRONIC RESEARCH ANNOUNCEMENTS OF THE AMERICAN MATHEMATICAL SOCIETY

Volume 10, Pages 142-154 (December 10, 2004)

S $1079-6762(04) 00139-8$

\title{
LOCAL RIGIDITY OF ACTIONS OF HIGHER RANK ABELIAN GROUPS AND KAM METHOD
}

\author{
DANIJELA DAMJANOVIĆ AND ANATOLE KATOK
}

(Communicated by Gregory Margulis)

\begin{abstract}
We develop a new method for proving local differentiable rigidity for actions of higher rank abelian groups. Unlike earlier methods it does not require previous knowledge of structural stability and instead uses a version of the KAM (Kolmogorov-Arnold-Moser) iterative scheme. As an application we show $\mathcal{C}^{\infty}$ local rigidity for $\mathbb{Z}^{k}(k \geq 2)$ partially hyperbolic actions by toral automorphisms. We also prove the existence of irreducible genuinely partially hyperbolic higher rank actions by automorphisms on any torus $\mathbb{T}^{N}$ for any even $N \geq 6$.
\end{abstract}

\section{INTRODUCTION}

1.1. Old and new approaches to rigidity. In this paper we introduce a new method of proving rigidity for smooth actions of higher rank abelian groups (i.e. $\mathbb{Z}^{k}$ and $\mathbb{R}^{k}$ for $k \geq 2$ ) on compact manifolds.

1.1.1. The Katok-Spatzier method. The old method introduced in [14] can be very briefly described as follows. One considers an action with sufficiently strong hyperbolic properties. The $C^{0}$ orbit structure of such an action is rigid (structural stability, Hirsch-Pugh-Shub theory, [5]). Moreover, the $C^{0}$ (orbit) conjugacy is unique transversally to the orbits. The method then consists in establishing a priori regularity of the conjugacy. The central idea is that locally in the phase space there are invariant geometric structures on invariant foliations for both the original action and a perturbed action. The simplest example of such a structure is affine (local linearization); in general the structure still has a finite-dimensional Lie group of automorphisms. Such a structure appears due to the fact that some elements of the action contract the foliations in question and this is true both in the rank one and the higher rank hyperbolic situation. Higher rank is crucial in showing that the conjugacy, which is a priori only continuous, intertwines the invariant structures and hence is smooth in the direction of each foliation. This in turn relies on the fact that in certain critical directions the unperturbed action acts by isometries on each leaf of the corresponding foliation. This in particular excludes the case of actions by automorphisms of the torus or a nil-manifold in the presence on nontrivial Jordan blocks.

Received by the editors September 19, 2004.

2000 Mathematics Subject Classification. Primary 37C85, 37C15, 58C15.

Key words and phrases. Local rigidity, group actions, KAM method, torus. Anatole Katok was partially supported by NSF grant DMS 0071339.

(C)2004 Danijela Damjanović and Anatole Katok Reverts to public domain 28 years from publication 
This method of establishing regularity of a conjugacy obtained from a different kind of reasoning appears as an ingredient in the proofs of local rigidity for actions of certain groups other than abelian [19, 3]. Notice that the earliest application of a version of this method can be found in [10] in the context of proving rigidity of some standard lattice actions on tori.

Both for the $\mathbb{R}^{k}$ actions and for the case of general lattice actions such as those considered in 3] there are certain residual directions left where regularity (and often even the existence) of the conjugacy has to be established separately. In the former case this follows from the cocycle rigidity which allows one to straighten out the time change 12 . Notice that cocycle rigidity extends to some partially hyperbolic actions 13 .

If one follows this approach for partially hyperbolic actions with semisimple linear part, one can establish rigidity of the neutral foliation for the unperturbed action (Theorem 4) and hence show that every small perturbation of the original action is differentiably conjugate to a perturbation along the neutral foliation. Since the latter is bigger than the orbit foliation the cocycle rigidity is not sufficient to finish the proof. On the other hand, there is no local (in the space) rigidity in this case. A priori there is no geometric structure on the neutral foliation preserved by individual elements of the perturbed action. Furthermore locally the higher rank condition does not help either. In other words, one can perturb the higher rank action locally (say, in a neighborhood of a periodic orbit) and change its topological structure in a dramatic way.

1.1.2. The new KAM-type method. In our new method we do not start from a conjugacy of low regularity. Instead we construct one of high regularity by an iterative process as a fixed point of a certain nonlinear operator. We use an iterative procedure, namely an adapted version of the KAM procedure motivated by the procedure used by Moser in 20] for commuting circle diffeomorphisms. Moser first noticed that commutativity along with simultaneous Diophantine condition was enough to provide a smooth solution to a certain overdetermined system of equations. Perhaps surprisingly, Moser's philosophy turned out to be applicable to a very different class of $\mathbb{Z}^{k}(k \geq 2)$ actions, namely actions by toral automorphisms.

The first key observation is that the linearized equation in this case is close to a twisted cocycle equation over the unperturbed action. Thus the "higher rank trick", which lies at the root of cocycle rigidity [12, 13], adapted to the twisted case, allows for the existence of an approximate solution of the linearized equation.

The second key observation is as follows. From the fact that the linearized equation is close to being the twisted cohomology equation we deduce that it is in fact close to a certain twisted cohomology equation for a cocycle over the unperturbed action. We construct this cocycle explicitly by adapting and developing a method from 8 .

In this paper we outline how this new approach works in the case of actions on the torus by automorphisms, affine maps and suspensions of such actions. We give precise formulation of the results and detailed description of the strategy of proofs.

As a byproduct of our approach we remove the restriction of semisimplicity for the unperturbed action. Our method works when the unperturbed action, whether hyperbolic or partially hyperbolic, has nontrivial Jordan blocks. A disadvantage of our method is the requirement that the perturbation is small in $C^{l}$ topology for a large $l$, while in the method of [14] perturbations small in $C^{1}$ topology are allowed. 
We also discuss examples of partially hyperbolic but not hyperbolic $\mathbb{Z}^{2}$ actions on the torus $\mathbb{T}^{N}$. We show that such genuinely partially hyperbolic actions do not exist on tori of dimension less than 6 . We also prove the existence of irreducible genuinely partially hyperbolic actions of $\mathbb{Z}^{2}$ on the torus of any even dimension starting from 6 , and in dimension 6 we give an explicit example. The existence of genuinely partially hyperbolic irreducible actions in any odd dimension is ruled out by the fact that any $A \in S L(N, \mathbb{Z})$ with irreducible characteristic polynomial and $N$ odd, is hyperbolic 23 . We also present a much simpler proof of this fact. However, reducible examples exist for all odd $N \geq 9$.

Detailed proofs can be found in [1].

There are other important classes of partially hyperbolic algebraic actions, for example restrictions of Weyl chamber flows to higher-rank subgroups or compact group extensions of Weyl chamber flows. The use of our methods to establish differentiable rigidity of partially hyperbolic actions of those types requires a substitution of the explicit Fourier analysis methods of the present paper by methods of the theory of unitary representations of semisimple Lie groups. In order to carry out constructions similar to those of special solutions of the second cohomology equation as in Section 2.3 one would need certain estimates of the decay for matrix coefficients, which are central for cocycle rigidity in [12, 14, as well as more specialized information about the structure of irreducible representations. This work is in progress.

We would like to thank Elon Lindenstrauss who pointed out an inaccuracy in an earlier version of [1].

1.2. Statement of results. For an action of a finitely generated group the $C^{n}$ topology is defined as the $C^{n}$ topology for any finite set of generators. For a smooth action of a connected Lie group it is defined similarly, with the vector fields generating the action of the Lie algebra playing the role of the generators.

For actions of discrete groups the notions of rigidity which we consider are summarized as follows.

Definition 1. An action $\alpha$ of a finitely generated discrete group $A$ on a manifold $M$ is $\mathcal{C}^{k, r, l}$ locally rigid if any sufficiently $\mathcal{C}^{r}$ small $\mathcal{C}^{k}$ perturbation $\tilde{\alpha}$ is $\mathcal{C}^{l}$ conjugate to $\alpha$, i.e., there exists a $\mathcal{C}^{l}$ close to identity diffeomorphism $\mathcal{H}$ of $M$ which conjugates $\tilde{\alpha}$ to $\alpha$ :

$$
\mathcal{H} \circ \alpha(g)=\tilde{\alpha}(g) \circ \mathcal{H}
$$

for all $g \in A$. The $\mathcal{C}^{\infty, 1, \infty}$ case is usually referred to as $\mathcal{C}^{\infty}$ local rigidity and the $\mathcal{C}^{1,1,0}$ case as $\mathcal{C}^{1}$ structural stability.

In the definitions for continuous groups such as $\mathbb{R}^{k}$ one has to allow a "time change", i.e. an automorphism $\rho$ of the group close to $i d$ such that instead of (1) one has

$$
\mathcal{H} \circ \alpha(\rho(g))=\tilde{\alpha}(g) \circ \mathcal{H} .
$$

A weaker notion in this case (and the one which is used in the definition of structural stability for $\mathbb{R}$ action) is foliation rigidity: one requires the diffeomorphism $\mathcal{H}$ to map an invariant foliation $\mathcal{F}$ (e.g. the orbit foliation $\mathcal{O}$ of the action $\alpha$ ) to the perturbed foliation $\mathcal{F}^{\prime}$ (e.g. the orbit foliation $\tilde{\mathcal{O}}$ of the action $\left.\tilde{\alpha}\right)$. 
Theorem 1. Let $\alpha: \mathbb{Z}^{k} \times \mathbb{T}^{N} \rightarrow \mathbb{T}^{N}$ be a $\mathcal{C}^{\infty}$ partially hyperbolic action of $\mathbb{Z}^{k}$ $(k \geq 2)$ by toral automorphisms with no nontrivial rank-one factors. Then there exists a constant $l=l(\alpha, N) \in \mathbb{N}$ such that for any $\mathcal{C}^{l}$ small $\mathcal{C}^{\infty}$ perturbation $\tilde{\alpha}$ of $\alpha$ there exists a $\mathcal{C}^{\infty}$ map $H: \mathbb{T}^{N} \rightarrow \mathbb{T}^{N}$ such that $\alpha \circ H=H \circ \tilde{\alpha}$, i.e., $\alpha$ is $\mathcal{C}^{\infty, l, \infty}$ locally rigid.

The constant $l$ in the above theorem depends on the dimension of the torus and the given linear action, and it will be precisely defined in the proof. However, for a certain class of actions this result can be improved so that the constant $l$ depend only on the dimension of the torus. By combining the method used by Katok and Spatzier for Anosov actions with semisimple linear parts [14 with the KAM procedure presented in this paper we obtain the following:

Theorem 2. There exists a constant $l=l(N)$ such that any partially hyperbolic action by automorphisms of the torus $T^{N}$ without rank one factors and with semisimple hyperbolic parts is $\mathcal{C}^{\infty, l, \infty}$ locally rigid.

Remark 1 . We note that the previous results extend to corresponding affine actions. While any affine map of the torus without eigenvalue one in its linear part has a fixed point and hence is conjugate to its linear part, the case of several commuting affine maps is more complicated (for examples of affine actions without fixed points see [6]). However, by passing to a subgroup of finite rank one reduces this case to the case of actions by automorphisms.

Remark 2. Another more or less direct extension of our results concerns suspensions of $\mathbb{Z}^{k}$ actions by toral automorphisms. Such a suspension is an $\mathbb{R}^{k}$ action and it allows obvious modifications by an automorphism of $\mathbb{R}^{k}$. The rigidity theorem in this case states that any small perturbation is differentiably conjugate to such a modification.

As for the existence of actions for which our method is essential we show:

Theorem 3. Genuinely partially hyperbolic (not hyperbolic) $\mathbb{Z}^{2}$ actions by ergodic toral automorphisms exist: on the torus of any even dimension $N \geq 6$ there are irreducible actions, while on any torus of any odd dimension $N \geq 9$ there are only reducible examples. There are no such actions on tori of dimension $N \leq 5$ and $N=7$.

Remark 3. One can also give similar conditions for the existence of genuinely partially hyperbolic actions of $\mathbb{Z}^{k}$ for $k>2$ by ergodic automorphisms. For example, an irreducible action of this kind exists in any even dimension starting from $2 k+2$.

In the rest of the paper we describe the strategy used for proving the above results. Complete proofs are contained in [1].

\section{Overview of KAM scheme and outline of the proof of Theorem 1}

2.1. Setting. Any matrix $A \in G L(N, \mathbb{Z})$ (i.e., a matrix with integer entries and determinant \pm 1 ) defines an automorphism of the torus $\mathbb{T}^{N}=\mathbb{R}^{N} / \mathbb{Z}^{N}$, which we also denote by $A$. Any such $A$ induces a decomposition of $\mathbb{R}^{N}$ into the expanding, neutral and contracting subspaces. The map $A$ of the torus induces a dual map given by the transpose matrix, which we also denote by $A$. Ergodicity of $A$ is equivalent to the fact that all nontrivial dual orbits are infinite, which in turn 
implies a well-known fact that $A$ is ergodic if and only if it has no roots of unity among the eigenvalues.

An action $\alpha: \mathbb{Z}^{k} \times \mathbb{T}^{N} \rightarrow \mathbb{T}^{N}$ by automorphisms of $\mathbb{T}^{N}$ is given by an embedding $\rho_{\alpha}: \mathbb{Z}^{k} \rightarrow G L(N, \mathbb{Z})$ so that

for each $g \in \mathbb{Z}^{k}$ and $x \in \mathbb{T}^{N}$.

$$
\alpha(g, x)=\rho_{\alpha}(g) x
$$

An action $\alpha^{\prime}$ is a rank-one factor if it is an algebraic factor (i.e., there exists an epimorphism $h: \mathbb{T}^{N} \rightarrow \mathbb{T}^{N}$ such that $\left.h \circ \alpha=\alpha^{\prime} \circ h\right)$ and is such that $\rho_{\alpha^{\prime}}\left(\mathbb{Z}^{k}\right)$ contains a cyclic subgroup of finite index.

An ergodic action $\alpha$ has no nontrivial rank-one factors if and only if $\rho_{\alpha}\left(\mathbb{Z}^{k}\right)$ contains a subgroup isomorphic to $\mathbb{Z}^{2}$ which consists of ergodic elements (see for example [22]).

Since we are interested in actions with no rank-one factors, we assume that the action $\alpha$ satisfies the following:

- There exist $g_{1}, g_{2} \in \mathbb{Z}^{2}$ with $A \stackrel{\text { def }}{=} \rho_{\alpha}\left(g_{1}\right)$ and $B \stackrel{\text { def }}{=} \rho_{\alpha}\left(g_{2}\right)$ such that any $A^{l} B^{k}$ for a nonzero $(l, k) \in \mathbb{Z}^{2}$ is ergodic. These will be referred to as ergodic generators.

- The action is genuinely partially hyperbolic, i.e., one can choose ergodic generators $A$ and $B$ so that they have common nontrivial neutral subspace and common hyperbolic subspace. We will assume that such a choice is made for $A$ and $B$. If the choice is not possible, the action is hyperbolic, and in that case we choose purely hyperbolic generators.

- We choose ergodic generators so that they have the same root spaces.

Now let $\tilde{\alpha}$ be a small perturbation of $\alpha$. The topology in which the perturbation is considered is a $C^{l}$ topology, where the lower bound for $l$ will be precisely defined in the proof. Our goal is to prove the existence of a $\mathcal{C}^{\infty}$ conjugacy, i.e., a map $H: \mathbb{T}^{N} \rightarrow \mathbb{T}^{N}$ such that $\tilde{\alpha} \circ H=H \circ \alpha$.

2.2. KAM scheme. The strategy we take is usual for KAM proofs. The unusual part is that while this strategy is known to have worked previously for elliptic situations, here we manage to carry it through in a global hyperbolic setting. The problem of finding a conjugacy is equivalent to solving the following nonlinear functional equation:

$$
\mathcal{N}(\tilde{\alpha}, H)=\tilde{\alpha} \circ H-H \circ \alpha=0 .
$$

Assuming the existence of a linear structure in the neighborhood of $i d$, we may look at the linearization of the operator $\mathcal{N}$ at $(\alpha, i d)$ :

$$
\begin{aligned}
\mathcal{N}(\tilde{\alpha}, H) & =\mathcal{N}(\alpha, i d)+D_{1} \mathcal{N}(\alpha, i d)(\tilde{\alpha}-\alpha)+D_{2} \mathcal{N}(\alpha, i d)(\Omega)+\operatorname{Res}(\tilde{\alpha}-\alpha, \Omega) \\
& =\tilde{\alpha}-\alpha+\alpha(\Omega)-(\Omega) \circ \alpha+\operatorname{Res}(\tilde{\alpha}-\alpha, \Omega)
\end{aligned}
$$

where $\Omega=H-i d$ and $\operatorname{Res}(\tilde{\alpha}-\alpha, \Omega)$ is quadratically small with respect to $\tilde{\alpha}-\alpha$ and $\Omega$. If one can find $H$ so that the linear part of the above expression vanish, i.e.,

$$
\alpha(H-i d)-(H-i d) \circ \alpha=-(\tilde{\alpha}-\alpha),
$$

then such an $H$ is a much better approximation to a solution to the equation $\mathcal{N}(\tilde{\alpha}, H)=0$ than the identity. After conjugating by $H$ and thus obtaining a smaller perturbation of the linear action, the linearization procedure and solving the linearized equation may be repeated for the new perturbation leading to an even better approximate solution. 
In particular, on the torus $\mathbb{T}^{N}$ any map can be lifted to the universal cover $\mathbb{R}^{N}$. For every $g \in \mathbb{Z}^{k}$, the lift of $\alpha(g)$ is a linear map of $\mathbb{R}^{N}$, i.e., a matrix with integer entries and with determinant \pm 1 , which is also denoted by $\alpha(g)$. The lift of $\tilde{\alpha}(g)$ is $\alpha(g)+\mathcal{R}(g)$, where $\mathcal{R}(g)$ is an $N$-periodic function for every $g$, i.e. $\mathcal{R}(g)(x+m)=\mathcal{R}(g)(x)$ for $m \in \mathbb{Z}^{N}$. The lift of $H$ has the form $i d+\Omega$ with an $N$-periodic $\Omega$.

In terms of $\Omega$ the conjugacy equation takes the following form:

$$
\alpha \Omega-\Omega \circ \alpha=-\mathcal{R} \circ(\text { id }+\Omega) .
$$

If $\Omega$ is an approximate solution for the corresponding linearized equation

$$
\alpha \Omega-\Omega \circ \alpha=-\mathcal{R},
$$

i.e., if it solves this equation with an error which is quadratically small with respect to $\mathcal{R}$, then the new perturbation defined as

$$
\tilde{\alpha}^{(1)} \stackrel{\text { def }}{=} H^{-1} \circ \tilde{\alpha} \circ H
$$

should be much closer to $\alpha$ than $\tilde{\alpha}$, i.e., the new error

$$
\mathcal{R}^{(1)} \stackrel{\text { def }}{=} \tilde{\alpha}^{(1)}-\alpha
$$

should be quadratically small with respect to the old error $\mathcal{R}$. The comparison of the two errors usually cannot be made in the same function space. The norm of the old error in some function space may only be comparable to the norm of the new error in some larger space. This is the "loss of regularity". In the proof that we present here there will be no actual loss of regularity at the end, namely, all the functions involved will be $\mathcal{C}^{\infty}$. We do however have a loss in the sense described above, and thus we work with the family of function spaces $\mathcal{C}^{r}(r \in \mathbb{N})$. Since we manage to obtain the loss of regularity which is fixed and since the family of spaces used admits smoothing operators, the iterative procedure can be set to converge to a solution of the nonlinear equation.

2.3. Individual step - the error estimate. The new error is

$$
\mathcal{R}^{(1)}=\tilde{\alpha}^{(1)}-\alpha=\left[\Omega \circ \tilde{\alpha}^{(1)}-\Omega \circ \alpha+\mathcal{R}(\mathrm{id}+\Omega)-\mathcal{R}\right]+[\mathcal{R}-(\alpha \circ \Omega-\Omega \circ \alpha)] .
$$

The error term in the first bracket comes from the linearization of the problem and is easy to estimate provided $\Omega$ is as small as $\mathcal{R}$. The difficulty lies in estimating the part of the error in the second bracket, namely solving the linearized equation (4) approximately, with an error quadratically small with respect to $\mathcal{R}$.

The linearized equation (4) actually consists of infinitely many equations corresponding to different elements of the action:

$$
\alpha\left(g_{i}\right) \Omega-\Omega \circ \alpha\left(g_{i}\right)=-\mathcal{R}\left(g_{i}\right)
$$

and we need a common (at least approximate) solution $\Omega$ to all the equations above. At this point two problems arise. The first is that $\mathcal{R}$ is not a cocycle over $\alpha$ and therefore this equation cannot be viewed as a cohomology equation over the linear action (thus one cannot immediately try to apply cocycle rigidity results for linear actions), and the second is that this is clearly an overdetermined problem in any case.

The answer to the second problem is that it is not necessary to find a common solution to all the equations above. Indeed, we can show that commutativity and ergodicity assumptions imply that it is enough to produce conjugacy $H$ for one 
ergodic generator. The same conjugacy will work for all other elements of the action. The key observation used here is discreteness of the centralizer for an ergodic partially hyperbolic automorphism of a torus. However, in general, it is not possible to produce a $\mathcal{C}^{\infty}$ conjugacy for a single map, since a single genuinely partially hyperbolic toral automorphism is not even structurally stable. In fact, we show that there are infinitely many obstructions to solving the linearized equation for one generator. In the case of an automorphism of a torus these obstructions may be represented as sums of Fourier coefficients along the dual orbits of the map with some weights added at each point, since our equations are twisted. Therefore, we consider two ergodic generators and reduce the problem of solving the linearized equation (4) to solving simultaneously the following system:

$$
\begin{aligned}
& A \Omega-\Omega \circ A=-\mathcal{R}_{A}, \\
& B \Omega-\Omega \circ B=-\mathcal{R}_{B},
\end{aligned}
$$

where $A$ and $B$ are ergodic generators. The system above splits further into several simpler systems using the fact that $A$ and $B$ commute and have common root spaces, i.e., (5) is reduced to several equations of the form

$$
\begin{aligned}
& J_{A} \Omega-\Omega \circ A=\Theta, \\
& J_{B} \Omega-\Omega \circ B=\Psi,
\end{aligned}
$$

where $J_{A}$ and $J_{B}$ are corresponding Jordan blocks. In particular, if $\lambda, \mu$ are simple eigenvalues of $A, B$ respectively, than we have

$$
\begin{aligned}
& \lambda \omega-\omega \circ A=\theta, \\
& \mu \omega-\omega \circ B=\psi,
\end{aligned}
$$

where $\theta$ and $\psi$ are $C^{\infty}$ functions on the torus.

2.3.1. Conditions for solvability of (5). Using the higher rank assumption, i.e. the existence of two elements of the action which generate an action by ergodic toral automorphisms, we show that all the obstructions to solving each individual equation above actually vanish provided that $\mathcal{R}$ satisfies a certain condition, which is satisfied if $\mathcal{R}$ is a twisted cocycle over the unperturbed action. The cocycle condition is (8) below.

The vanishing of the obstructions allows one to construct a distribution solution of the system (7), which can be proven to be $C^{\infty}$. Moreover, using the cone method of 24 it is possible to obtain an estimate for the $C^{r}$ norm of this solution with respect to the $C^{r+\sigma}$ norm of $\theta$ and $\psi$ with only a fixed loss of regularity. This loss $\sigma$ depends on the dimension $N$ and eigenvalues of $A$ and $B$. Obtaining estimates is not difficult when ergodic generators are hyperbolic. The existence of a nontrivial neutral direction requires the use of the fact that integer vectors cannot remain mostly in the neutral direction for too long. In fact, the time for which they remain mostly in the neutral direction is at most approximately logarithmic with respect to the norm of the vector. The precise statement which is used is that of Lemma 3 from Katznelson's paper [15]. Thus the decay of Fourier coefficients along the dual orbits of the action can be well estimated for all the orbits, and this implies the norm estimate of the solution.

More generally, allowing the existence of Jordan blocks, we show that there exists a $\mathcal{C}^{\infty}$ solution to (5I) if the following cocycle condition is satisfied by $\mathcal{R}$ :

$$
L\left(\mathcal{R}_{A}, \mathcal{R}_{B}\right) \stackrel{\text { def }}{=} \Delta^{B} \mathcal{R}_{A}-\Delta^{A} \mathcal{R}_{B} \equiv 0
$$


where $\Delta^{B} \mathcal{R}_{A} \stackrel{\text { def }}{=} \mathcal{R}_{A} \circ B-B \mathcal{R}_{B}$ and $\Delta^{A} \mathcal{R}_{B} \stackrel{\text { def }}{=} \mathcal{R}_{B} \circ A-A \mathcal{R}_{B}$

The precise statement is the following lemma proven in 1 .

Lemma 1. If $L\left(\mathcal{R}_{A}, \mathcal{R}_{B}\right) \equiv 0$, where $\mathcal{R}_{A}, \mathcal{R}_{B}$ are $\mathcal{C}^{\infty}$ maps described before, then the equations (5),

$$
\begin{aligned}
\Delta^{A} \Omega & =\mathcal{R}_{A}, \\
\Delta^{B} \Omega & =\mathcal{R}_{B}
\end{aligned}
$$

have a common $\mathcal{C}^{\infty}$ solution satisfying

$$
\|\Omega\|_{\mathcal{C}^{r}} \leq C_{r}\left\|\mathcal{R}_{A}, \mathcal{R}_{B}\right\|_{\mathcal{C}^{r+\sigma}}
$$

for any $r>0$ and $\sigma>M_{1}=\max \left\{N+1, M_{0}(A, B)\right\}$, where $M_{0}$ is the logarithm of the spectrum width for $A$ or for $B$, whichever is greater. In particular, if the perturbation $\tilde{\alpha}$ is in the neutral direction only, then $M_{0}=0$.

2.3.2. Approximating an almost solvable system by a solvable one. Even though the error term $\mathcal{R}$ may not satisfy the cocycle condition (88) over the unperturbed action, the fact that it satisfies the twisted cocycle condition over the perturbed action implies that it satisfies the cocycle condition for the unperturbed action up to an error which is quadratically small with respect to $\mathcal{R}$. More precisely, it is easy to show that if $\tilde{\alpha}=\alpha+\mathcal{R}$ is a commutative action, then $L\left(\mathcal{R}_{A}, \mathcal{R}_{B}\right)$ is quadratically small in the $\mathcal{C}^{0}$ norm with respect to $\mathcal{R}_{A}, \mathcal{R}_{B}$. This leads us to the key step: we prove that any almost cocycle over $\alpha$ is close to a cocycle over $\alpha$. This requires finding a projection of $\mathcal{R}$ to the space of cocycles over $\alpha$. What makes this part of the problem difficult is the need to find a projection such that the $C^{r}$ norm of the difference is quadratically small with respect to some $C^{r+\sigma}$ norm of $\mathcal{R}$, where $\sigma$ is a fixed constant independent of individual steps of the scheme. We construct this projection effectively. By reducing the problem again to simpler equations the core of the problem is to obtain tame solutions $\tilde{\theta}$ and $\tilde{\psi}$ to an equation of the kind

$$
(\mu \tilde{\theta}-\tilde{\theta} \circ B)-(\lambda \tilde{\psi}-\tilde{\psi} \circ A)=\varphi
$$

assuming that $\theta$ and $\psi$ satisfy the same equation with $\varphi$ on the right hand side, i.e. assuming that the "double" obstructions for $\varphi$ vanish.

The approach to this part of the problem is as follows: the fact that $\psi, \theta$ and $\varphi$ satisfy the equation above implies that the obstructions for $\psi$ with respect to $B$, i.e., sums of the Fourier coefficients of $\psi$ along the dual orbits of $B$ (even if they do not vanish) are actually small of the order of $\varphi$. So the strategy is to construct $\tilde{\psi}$ by defining its Fourier coefficients to be zero on each dual orbit except at the minimal point on the orbit where we assign the Fourier coefficient of $\tilde{\psi}$ to be the obstruction of $\psi$ along that orbit. Thus $\tilde{\psi}$ and $\psi$ have the same obstructions and their difference has vanishing obstructions so that the linearized equation for the difference $\psi-\tilde{\psi}$ can be solved. The crucial part is that the estimates are needed for the Fourier coefficients of $\tilde{\psi}$, i.e., for the obstructions of $\psi$ with respect to $\varphi$. This involves estimating the decay of Fourier coefficients for $\varphi$ along the "double" dual orbits, i.e., the dual orbits of the action, rather than for only one generator. At first glance this may pose a problem, since the expanding and contracting directions for two generators may mix obstructing exponential decay. In fact, there may be a situation where some (infinitely many) elements have eigenvalues outside the unit circle but still very close to it. However, due to the higher rank assumption if this happens, there must be an extra direction which grows. For, otherwise one would 
have integer matrices close to the identity, which is not possible unless they are the identity. The existence of this extra direction, along with the fact that it ought to be irrational, implies (using Katznelson's lemma again) that there is a sufficient exponential decay, enough to imply norm estimates for the "double" obstructions for $\varphi$ and thus for "single" obstructions for $\psi$.

We further show that this procedure can be carried out in the case where there are nontrivial Jordan blocks and that the solution is tame. More precisely, we obtain:

Lemma 2. For two $\mathcal{C}^{\infty}$ maps $\mathcal{R}_{A}, \mathcal{R}_{B}$ with $L\left(\mathcal{R}_{A}, \mathcal{R}_{B}\right)=\Phi$ there exist splittings $\mathcal{R}_{A}=\dot{\mathcal{R}}_{A}+\tilde{\mathcal{R}}_{A}$ and $\mathcal{R}_{B}=\dot{\mathcal{R}}_{B}+\tilde{\mathcal{R}}_{B}$ such that

$$
\begin{gathered}
L\left(\dot{\mathcal{R}}_{A}, \dot{\mathcal{R}}_{B}\right)=0, \quad L\left(\tilde{\mathcal{R}}_{A}, \tilde{\mathcal{R}}_{B}\right)=\Phi, \\
\left\|\dot{\mathcal{R}}_{A}, \dot{\mathcal{R}}_{B}\right\|_{\mathcal{C}^{r}} \leq C_{r}\|\mathcal{R}\|_{\mathcal{C}^{r+\sigma}}
\end{gathered}
$$

for any $r>0$ and $\sigma>M^{1}=(N+1) \max \left\{1, M_{0}\right\}$. Furthermore,

$$
\left\|\tilde{\mathcal{R}}_{A}, \tilde{\mathcal{R}}_{B}\right\|_{\mathcal{C}^{r}} \leq C_{r}\|\Phi\|_{\mathcal{C}^{r+\sigma}}
$$

for any $r>0$ and $\sigma>M$, where the constant $M$, similarly to $M_{1}$ and $M^{1}$, depends only on the dimension of the torus and the action generated by $A$ and $B$, and also reduces to $N+1$ in the case when the action is perturbed in the neutral direction only.

Remark 4. The constant $M$ is precisely defined in the proof of the above lemma in Section 3.3 of [1]. It involves a constant which depends on the action generated by $A$ and $B$ and characterizes the exponential growth along dual orbits of the action with respect to certain projections.

The above discussion implies that there exists a tame (with respect to $\mathcal{R}$ ), and tamely approximate (the error is quadratically small with respect to $\mathcal{R}$ ) solution to the linearized equation (5).

2.3.3. Smoothing. The loss of a fixed number of derivatives at each step of the iteration process can be overcome by introducing a family of smoothing operators: $\left\{S_{J}, J \in \mathbb{N}\right\}$. Then instead of approximately solving (5) we approximately solve the following system:

$$
\begin{aligned}
& A \Omega-\Omega \circ A=-S_{J} \mathcal{R}_{A}, \\
& B \Omega-\Omega \circ B=-S_{J} \mathcal{R}_{B} .
\end{aligned}
$$

For a $\mathcal{C}^{\infty}$ function $f$ with Fourier series $f=\sum_{n} \hat{f}_{n} \chi_{n}$ we define $S_{J} f$ as

$$
S_{J} f \stackrel{\text { def }}{=} \sum_{|n|<J} \hat{f}_{n} \chi_{n} .
$$

Then the smoothing operators satisfy

$$
\left\|S_{J} f\right\|_{\mathcal{C}^{a+b}} \leq J^{b+\sigma}\|f\|_{\mathcal{C}^{a}}
$$

where $a>b>0$ and $\sigma>N+1$. Also

$$
\left\|\left(I-S_{J}\right) f\right\|_{\mathcal{C}^{a-b}} \leq C J^{-b+\sigma}\|f\|_{\mathcal{C}^{a}}
$$

for $a>b>\sigma>N+1$. These simple smoothing operators are convenient in our setting since they behave well with respect to the operator $L$. Namely, we have

$$
L\left(S_{J} f, S_{J} g\right)=S_{\frac{J}{\xi}}(L(f, g))+\mathcal{F}_{>\frac{J}{\xi}},
$$


where $\xi$ is a constant depending on $A$ and $B$ and the last term in the expression consists of the part of the Fourier series for $f$ and $g$ with terms $|n|>\frac{J}{\xi}$.

This implies the following estimate:

$$
\left\|L\left(S_{J} f, S_{J} g\right)\right\|_{\mathcal{C}^{r}} \leq J^{2 \sigma}\|L(f, g)\|_{\mathcal{C}^{r}}+C J^{-b+\sigma}\|f, g\|_{\mathcal{C}^{r+b}}
$$

for any $b>\sigma>N+1$.

2.3.4. Estimate for the error. At each step of the iterative scheme we first choose an appropriate smoothing operator $S_{J}$. In order to approximately solve (9) we use Lemma 2 to obtain the splitting

$$
\begin{aligned}
& S_{J} \mathcal{R}_{A}=\overline{S_{J} \mathcal{R}_{A}}+\widetilde{S_{J} \mathcal{R}_{A}}, \\
& S_{J} \mathcal{R}_{B}=\overline{S_{J} \mathcal{R}_{B}}+\widetilde{S_{J} \mathcal{R}_{B}},
\end{aligned}
$$

so that $L\left(\overline{S_{J} \mathcal{R}_{A}}, \overline{S_{J} \mathcal{R}_{B}}\right)=0$. Here, we use the bar notation $\bar{f}$ instead of the $\operatorname{dot}$ notation $\dot{f}$ of Lemma 2 Now Lemma 1 implies that the system

$$
\begin{aligned}
& A \Omega-\Omega \circ A=-\overline{S_{J} \mathcal{R}_{A}}, \\
& B \Omega-\Omega \circ B=-\overline{S_{J} \mathcal{R}_{B}}
\end{aligned}
$$

has an approximate solution $\Omega$ such that

$$
\|\Omega\|_{\mathcal{C}^{r}} \leq C_{r} J^{3 \sigma}\left\|\mathcal{R}_{A}, \mathcal{R}_{B}\right\|_{\mathcal{C}^{r}}
$$

Then we define $H \stackrel{\text { def }}{=} i d+\Omega$ (since $\Omega$ is made small throughout the iteration, $H$ is invertible) and

$$
\tilde{\alpha}^{(1)} \stackrel{\text { def }}{=} H^{-1} \circ \tilde{\alpha} \circ H \text {. }
$$

The new error is equal to

$$
\mathcal{R}^{(1)} \stackrel{\text { def }}{=} \tilde{\alpha}^{(1)}-\alpha
$$

and it has two parts:

- The error coming from solving the linearized equation only approximately,

$$
E_{1}=\widetilde{S_{J} \mathcal{R}}+\left(I-S_{J}\right) \mathcal{R} .
$$

- The standard error coming from the linearization,

$$
E_{2}=\Omega \circ \tilde{\alpha}^{(1)}-\Omega \circ \alpha+\mathcal{R}(i d+\Omega)-\mathcal{R} .
$$

We estimate both parts of the error using the estimates obtained after approximately solving the linearized equation and bounds for the smoothing operators. The new error can be estimated as

$$
\left\|\mathcal{R}^{(1)}\right\|_{\mathcal{C}^{0}} \leq C J^{3 \sigma}\|\mathcal{R}\|_{\mathcal{C}^{1}}\|\mathcal{R}\|_{\mathcal{C}^{0}}+C J^{-l+2 \sigma}\|\mathcal{R}\|_{\mathcal{C}^{l}}
$$

for any $l>2 \sigma$. From $\tilde{\alpha}^{(1)}=H^{-1} \circ \tilde{\alpha} \circ H$, using the fact that $\Omega$ is $N$-periodic and satisfies the estimate (10), we have

$$
\left\|\mathcal{R}^{(1)}\right\|_{\mathcal{C}^{l}} \leq C_{l} J^{3 \sigma}\left(1+\|\mathcal{R}\|_{\mathcal{C}^{l}}\right) .
$$


2.4. Setting the iteration and convergence. To set up the iterative process let

$$
\mathcal{R}^{(0)}=\mathcal{R} ; \quad \tilde{\alpha}^{(0)}=\tilde{\alpha} ; \quad H^{(0)}=i d .
$$

Construct $\mathcal{R}^{(n)}$ inductively for every $n$ : for $\mathcal{R}^{(n)}$ we choose an appropriate $J_{n}$ to obtain $S_{J_{n}} \mathcal{R}^{(n)}$, which produces a new $\Omega^{(n)}$ after approximately solving the linearized equation. Then we have

$$
\begin{aligned}
H^{(n)}=i d & +\Omega^{(n)}, \quad \tilde{\alpha}^{(n+1)}=\left(H^{(n)}\right)^{-1} \circ \tilde{\alpha}^{(n)} \circ H^{(n)}, \quad \mathcal{R}^{(n+1)}=\tilde{\alpha}^{(n+1)}-\alpha \\
\tilde{\alpha}^{(n+1)} & =\left(H^{(n)}\right)^{-1} \circ\left(H^{(n-1)}\right)^{-1} \circ \cdots \circ\left(H^{(0)}\right)^{-1} \circ \tilde{\alpha} \circ H \circ \cdots \circ H^{(n)} \\
& =\mathcal{H}_{n}^{-1} \circ \tilde{\alpha} \circ \mathcal{H}_{n} .
\end{aligned}
$$

To ensure the convergence of the process we set

$$
\begin{gathered}
\left\|\mathcal{R}^{(n)}\right\|_{\mathcal{C}^{0}}<\varepsilon_{n}=\varepsilon^{\left(k^{n}\right)}, \quad\left\|\mathcal{R}^{(n)}\right\|_{\mathcal{C}^{l}}<\varepsilon_{n}^{-1}, \\
\left\|\Omega^{(n)}\right\|_{\mathcal{C}^{1}}<\varepsilon_{n}^{1 / 2}, \quad J_{n}=\varepsilon_{n}^{-\frac{1}{3(3 \sigma+2)}},
\end{gathered}
$$

where $k=\frac{4}{3}$. At this point we fix $l: l=23 \sigma+15$, where $\sigma=\sigma(A, B, N)=$ $\left[\max \left\{M, M^{1}, M_{1}\right\}\right]+1$ is a constant for which all the estimates obtained above hold.

Convergence of the process is then proven by induction, using the estimates already obtained. Namely, for sufficiently small $\|\mathcal{R}\|_{\mathcal{C}^{0}}$ and $\|\mathcal{R}\|_{\mathcal{C}^{1}}$ the process converges to a solution $\Omega \in \mathcal{C}^{1}$ with $\|\Omega\|_{\mathcal{C}^{1}}<\frac{1}{4}$. Then, using interpolation inequalities, we show that $\Omega$ is $\mathcal{C}^{\infty}$.

\section{Outline of the Proof of Theorem 2}

Theorem 4 (Foliation rigidity). The neutral foliation $\mathcal{N}$ of a $C^{\infty}$ action $\alpha$ by partially hyperbolic automorphisms of $\mathbb{T}^{N}$ without rank one factors and with semisimple hyperbolic parts is $C^{\infty}$ rigid, i.e., for any $C^{\infty}$ foliation $\mathcal{N}^{\prime}$ sufficiently close to $\mathcal{N}$ in the $C^{1}$ topology there exists a $C^{\infty}$ diffeomorphism $h: \mathbb{T}^{N} \rightarrow \mathbb{T}^{N}$ such that $h \mathcal{N}=\mathcal{N}^{\prime}$.

This theorem is proven exactly like Theorem 1 and Theorem 6 in [14. In fact, a more general statement is true concerning foliation rigidity for a broad class of homogeneous partially hyperbolic actions [14.

This reduces the local rigidity problem for an action with the semisimple linear part to showing that a sufficiently small perturbation along the leaves of the neutral foliation of a given linear action is smoothly conjugate to the original action. This is a special case of Theorem 1. But in this special situation the estimates obtained for the solution of the corresponding linearized equation are much better. Namely, since the action is perturbed only in the neutral direction, the linearized equation splits into several equations of the form (7) or (6) with $|\lambda|=|\mu|=1$. The constants that describe the loss of regularity turn out to be at most $N+2$ in this case. Therefore, in the iterative proof for perturbations in the neutral direction it is sufficient to choose $l=l(N)=23 N+61$ for the convergence of the successive approximations to a $\mathcal{C}^{\infty}$ conjugacy.

Combined with the foliation rigidity result where the perturbation needs to be only $\mathcal{C}^{1}$ small, this implies $\mathcal{C}^{\infty, l, \infty}$ local rigidity for an action with the semisimple linear part, where $l$ is defined as above and hence depends only of the dimension of the torus. 


\section{EXISTENCE OF GENUINELY PARTIALLY HYPERBOLIC ACTIONS}

To prove the statement of Theorem 3 we first show case by case that there are no examples of genuinely partially hyperbolic $\mathbb{Z}^{k}$ actions (irreducible or reducible) for $N \leq 5$. In [23] it is proven that there are no irreducible automorphisms in odd dimensions with nontrivial neutral direction. In Section 6.2 of [1] we give a simple proof of this fact. Then we show that there are irreducible examples of such actions in any even dimension $N \geq 6$. For that we use matrices with recurrent characteristic polynomials. By taking direct products of these examples with hyperbolic actions we obtain reducible examples in any odd dimension $N \geq 9$. For example on the 9dimensional torus, in the construction above take $n_{1}=3, n_{2}=6$, let $A_{1,2}$ and $A_{2,2}$ be commuting matrices that give an irreducible action in even dimension (we give explicit examples of those), and for $A_{1,1}$ and $A_{2,1}$ choose any two $3 \times 3$ commuting hyperbolic totally real integer matrices (for various examples see [9]).

\section{REFERENCES}

[1] D. Damjanovic and A. Katok, Local rigidity of partially hyperbolic actions of $\mathbb{Z}^{k}$ and $\mathbb{R}^{k}, k \geq$ 2. I. KAM method and actions on the torus, Preprint, 2004, http://www.math.psu.edu/katok_a/papers.html

[2] M. Einseidler, A. Katok, and E. Lindenstrauss, Invariant measures and the set of exceptions in Littlewood's conjecture, Ann. Math., to appear.

[3] D. Fisher and G.A. Margulis, Local rigidity for affine actions of higher rank Lie groups and their lattices, Preprint, 2004.

[4] M. Guysinsky and A. Katok, Normal forms and invariant geometric structures for dynamical systems with invariant contracting foliations, Math. Res. Lett. 5 (1998), no. 1-2, 149-163. MR:1618331 (99b:58215)

[5] M. Hirsch, C. Pugh, and M. Shub, Invariant manifolds, Lecture Notes in Mathematics 583, Springer-Verlag, Berlin, 1977. MR 0501173 (58:18595)

[6] S. Hurder, Affine Anosov actions, Michigan Math. J. 40 (1993), no. 3, 561-575. MR1236179 $(94 \mathrm{~g}: 58165)$

[7] A. Katok and B. Hasselblatt, Introduction to the modern theory of dynamical systems, Encyclopedia of Mathematics and Its Applications 54, Cambridge University Press, 1995. MR.1326374 (96c:58055)

[8] A. Katok and S. Katok, Higher cohomology for abelian groups of toral automorphisms, Ergod. Th. Dynam. Sys. 15 (1995), 569-592. MR1336707 (96k:58167)

[9] A. Katok, S. Katok, and K. Schmidt, Rigidity of measurable structure for $\mathbb{Z}^{d}$ actions by automorphisms of a torus, Comm. Math. Helv. 77 (2002), 718-745. MR1949111 (2003h:37007)

[10] A. Katok and J. Lewis, Local rigidity of certain groups of toral automorphisms, Israel J. Math. 75 (1991), 203-241. MR,1164591 (93g:58076)

[11] A. Katok and J. Lewis, Global rigidity results for lattice actions on tori and new examples of volume-preserving action, Israel J. Math. 93 (1996), 253-280. MR1380646 (96k:22021)

[12] A. Katok and R. Spatzier, First cohomology of Anosov actions of higher rank abelian groups and applications to rigidity, Publ. Math. I.H.E.S., 79 (1994), 131-156. MR1307298 (96c:58132)

[13] A. Katok and R. Spatzier, Subelliptic estimates of polynomial differential operators and applications to rigidity of abelian actions. Math. Res. Lett. 1 (1994), no. 2, 193-202. MR1266758 (95b:35042)

[14] A. Katok and R. Spatzier, Differential rigidity of Anosov actions of higher rank abelian groups and algebraic lattice actions, Proc. Steklov Inst. Math. 216 (1997), 287-314. MR1632177 (99i:58118)

[15] Y. Katznelson, Ergodic automorphisms on $\mathbb{T}^{n}$ are Bernoulli shifts, Israel J. Math. 10 (1971), 186-195. MR0294602 (45:3672)

[16] V. F. Lazutkin, KAM theory and semiclassical approximations to eigenfunctions, SpringerVerlag, Berlin, 1993. MR,1239173 (94m:58069) 
[17] R. de la Llave, Tutorial on KAM theory. Smooth ergodic theory and its applications, Proc. Sympos. Pure Math. 69, Amer. Math. Soc., Providence, RI, 2001, pp. 175-292. MR 1858536 (2002h:37123)

[18] R. Mañè, A proof of the $\mathcal{C}^{1}$ stability conjecture, Publ. Math. I.H.E.S. 66 (1988), 161-210. MR 0932138 (89e:58090)

[19] G. A. Margulis and N. Qian, Rigidity of weakly hyperbolic actions of higher real rank semisimple Lie groups and their lattices, Ergod. Th. Dynam. Sys. 21 (2001), 121-164. MR1826664 (2003a:22019)

[20] J. Moser, On commuting circle mappings and simultaneous Diophantine approximations, Math. Z. 205 (1990), 105-121. MR1069487 (92e:58120)

[21] J. Robbin, A structural stability theorem, Ann. Math. 94 (1971), 447-493. MR0287580 $(44: 4783)$

[22] A. Starkov, The first cohomology group, mixing, and minimal sets of the commutative group of algebraic actions on a torus, Dynamical systems, 7, J. Math. Sci. (New York) 95 (1999), no. 5, 2576-2582. MR:1712745 (2000m:37005)

[23] F. Rodrigues Hertz, Stable ergodicity of certain linear automorphisms of the torus, Ann. Math. (2004), to appear.

[24] W. Veech, Periodic points and invariant pseudomeasures, Ergod. Th. Dynam. Sys. 6 (1986), 449-473. MR0863205 (87m:58096)

Department of Mathematics, The Pennsylvania State University, University Park, PA 16802

Current address: Erwin Schroedinger Institute, Boltzmanngasse 9, A-1090 Vienna, Austria

E-mail address: damjanov@math.psu.edu

$U R L:$ http://www.math.psu.edu/damjanov

Department of Mathematics, The Pennsylvania State University, University Park, PA 16802

E-mail address: katok_a@math.psu.edu

URL: http://www.math.psu.edu/katok_a 